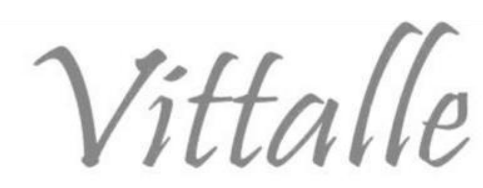

\title{
A trajetória de um programa de extensão no contexto de um hospital universitário federal
}

\author{
Ana Luiza Muccillo-Baisch*, Cristiane Guimarães Fonseca dos Santos, Fernando \\ Corrêa Senna, Gicelda Mara Ferreira da Silva, Liziane Göebel Casarin Jaekel, Maria \\ Cristina Flores Soares, Maria Cristine Igansi da Cunha, Maria da Penha da Rosa \\ Silveira, Quelen Iane Garlet, Raquel Soares Bonatto, Sara Silva Fernandes, Seiko \\ Nomiyama
}

Centro Regional de Estudos, Prevenção e Recuperação de Dependentes Químicos - CENPRE, Instituto de Ciências Biológicas, Universidade Federal do Rio Grande - FURG, Rio Grande, RS, Brasil

Histórico do Artigo
Recebido em:
06/03/2020
Aceito em:
21/06/2020
Palavras-chave:
Programa de extensão;
prevenção; droga
adição;
multidisciplinariedade;
tratamento
ambulatorial; saúde
mental

Histórico do Artigo

$21 / 06 / 2020$

Palavras-chave:

Programa de extensão; prevenção; droga adição;

tratamento mental

Keywords:

Extension program; prevention; drug addiction; multidisciplinary; outpatient treatment; mental health

\begin{abstract}
RESUMO
Este trabalho pretende mostrar como foi idealizado e como funciona na atualidade o Centro Regional de Estudos, Prevenção e Recuperação de Dependentes Químicos (CENPRE), um programa de extensão permanente da Universidade Federal do Rio Grande (FURG). O CENPRE surgiu a partir das atividades extensionistas desenvolvidas pelo antigo Departamento de Ciências Fisiológicas (DCF), hoje Instituto de Ciências Biológicas (ICB), e foi idealizado para cumprir os objetivos das diretrizes de prevenção ao uso de substâncias psicoativas. Inserido no contexto do Hospital universitário da FURG, o CENPRE conduz suas atividades utilizando uma abordagem interdisciplinar, composta pelo trabalho de um psiquiatra, três psicólogos, duas enfermeiras, um terapeuta familiar, uma assistente social e uma farmacêutica. Adicionalmente, este centro de pesquisas se mantém integrado à comunidade acadêmica e à população em geral, com vistas à promoção da saúde, à prevenção e ao tratamento de transtornos relacionados ao uso de substâncias psicoativas. O CENPRE se consolidou como um centro de referência e apoio à formação de recursos humanos por meio de ações educativas e atividades de ensino; além de fomentar a pesquisa por intermédio de estudos epidemiológicos em comunidades locais e regionais.
\end{abstract}

The trajectory of an extension program in the context of a federal university hospital

\section{ABSTRACT}

The present work aims to show the Regional Center for Studies, Prevention and Recovery of Chemical Dependents (CENPRE) history, including the scenario where it was conceived and how the Center works today: a permanent extension program at the Federal University of Rio Grande (FURG). CENPRE is originated from the extension activities developed by the former Department of Physiological Sciences (DCF), today the Institute of Biological Sciences (ICB), and it was designed to meet the objectives of psychoactive substances abuse prevention guidelines. Inserted in the context of the FURG University Hospital, CENPRE seeks to conduct its activities using an interdisciplinary approach, which is composed by the work of a psychiatrist, three psychologist, two nurses, a family counselor, a social worker, and pharmacist. Furthermore, this research Center is integrated with the academic community and the population in general and offers services of health promotion, and the outpatient treatment of psychoactive substancesrelated disorders. CENPRE is established as a reference and support center for human resources training through educational and teaching activities, besides promoting research through epidemiological studies in local and regional communities.

\section{Contextualização histórica}

Em 1988, Estados-membros da Organização das Nações Unidas (ONU) decidiram fortalecer as ações para combater o tráfico de drogas. A Convenção de 1988 teve como

\footnotetext{
*Autor correspondente: anabaisch@ gmail.com (Muccillo-Baisch A.L.)
} 
objetivo promover a cooperação entre os Estados para tratar de forma mais eficaz o tráfico de drogas, acabar com os lucros de organizações criminosas através da produção de drogas ilícitas e do tráfico e fornecer novas ferramentas aos governos. Na mesma convenção os países firmaram o compromisso a adotarem medidas efetivas nas áreas de prevenção, tratamento e reabilitação (1).

No Brasil, em 1987, por iniciativa da antiga Secretaria Nacional Antidrogas, atual Secretaria Nacional de Políticas sobre Drogas (SENAD) e com a parceria do Centro Brasileiro de Informações sobre Drogas (CEBRID) da Universidade Federal de São Paulo, iniciou-se uma série de levantamentos com o intuito de conhecer a prevalência e os padrões de consumo de drogas e suas consequências entre estudantes brasileiros dos ensinos fundamental e médio das redes pública e privada (2). Em 2002, o Ministério da Saúde constituiu os Centros de Atenção Psicossocial (CAPS) por meio da Portaria no 336/2002 e instituiu o Programa Nacional de Atenção Comunitária Integrada aos Usuários de Álcool e Outras Drogas, para promover a reabilitação e a reinserção social dos usuários (3). A constituição desses dispositivos propôs um modelo de atenção biopsicossocial com foco não apenas nas questões orgânicas e psíquicas do usuário/dependente químico, mas no seu contexto social, político, econômico e cultural (4-5).

No ano de 2005, o Conselho Nacional Anti Drogas (CONAD) aprovou a Política Nacional sobre Drogas (6) priorizando a prevenção e entendendo que esta intervenção é mais eficaz e menos onerosa para a sociedade. Também considerou a redução de danos uma estratégia inserida na prevenção e garantiu o direito dos pacientes receberem tratamento adequado. No ano seguinte, foi instituído o Sistema Nacional de Políticas Públicas sobre Drogas (SISNAD), que estabeleceu estratégias para prevenção do uso indevido de substâncias, atenção e reinserção social de dependentes químicos (7).

Diversas estratégias foram desenvolvidas e culminaram, em 2011, na instituição da Rede de Atenção Psicossocial (RAPS) (8), que veio com o propósito de garantir a articulação e a integração das redes de atenção à saúde (implantadas nos territórios), qualificando o cuidado por meio do acolhimento, acompanhamento contínuo, atenção às urgências e atendimento psicossocial da população, tanto com transtornos mentais quanto com necessidades que decorrem do uso prejudicial de drogas (9-10).

No Rio Grande do Sul, o Conselho Estadual de Entorpecentes (CONEN) foi instituído em 1996 (11), e tinha como atribuição formular políticas públicas de educação preventiva, tratamento, assistência e recuperação de dependes químicos. Mas apenas em 2011 se registra um importante marco político: a instituição do Sistema Estadual de Políticas Públicas sobre Drogas (12). Pela primeira vez, a lei estabelece princípios de respeito aos direitos humanos, à diversidade, às particularidades sociais, culturais e comportamentais, e o combate à discriminação e à estigmatização social.

O município do Rio Grande, RS, iniciou sua própria caminhada na prevenção ao uso de drogas lícitas e ilícitas quando regimentou as normas para a venda da cola de sapateiro, a proibição do fumo em repartições públicas municipais e a proibição da venda e do consumo de bebidas alcoólicas nos estabelecimentos de ensino do município (Pietro, 2012) (13). Em abril de 2014 institui a Política municipal sobre drogas, regulamenta o fundo municipal de políticas sobre drogas e cria a Coordenadoria da política municipal sobre drogas (14).

Nesse cenário da Reforma Psiquiátrica, a Universidade Federal do Rio Grande (FURG), com o protagonismo do Setor de Farmacologia do antigo Departamento de Ciências Fisiológicas (DCF), hoje Instituto de Ciências Biológicas (ICB), iniciou o desenvolvimento de atividades extensionistas dentro do Projeto Educativo e Preventivo sobre Drogas Psicotrópicas. Este projeto ficou conhecido como Projeto Drogas e foi concebido em 1989, tendo como objetivos a educação e a prevenção ao uso de 
substâncias psicoativas (SPA).

Nessa época já existia uma grande demanda na região para ações educativas em escolas. As necessidades apontadas pelos grupos populacionais, pelo momento histórico e pela comunidade científica, culminaram em um Projeto Preventivo, cujos objetivos eram conhecer a realidade do consumo de SPA entre os escolares dos ensinos fundamental e médio; propor um programa educativo e preventivo para as escolas; estimular as escolas a criarem suas próprias ações prevencionistas; e promover a capacitação dos professores (15).

Assim, entre os anos de 1989 e 1990, foi realizado o primeiro levantamento epidemiológico entre os escolares dos ensinos fundamental e médio nos municípios de Rio Grande, Santa Vitória do Palmar e São José do Norte, com testagem do instrumento e aplicação de 11.817 questionários nos três municípios (www.cenpre.furg.br).

Ainda como parte do Projeto Preventivo, considerando-se a carência de serviços especializados para tratamento de dependentes químicos na região, foi criado em agosto de 1999, o Centro Regional de Estudos, Prevenção e Recuperação de Dependentes Químicos (CENPRE), que iniciou suas atividades na Ala Azul do Hospital Universitário Dr. Miguel Riet Corrêa Jr. (HU/FURG), inaugurando o serviço de tratamento e de organização do seu banco de dados e mantendo ativo o serviço de prevenção já existente (15).

O CENPRE se consolidou como um programa permanente de extensão universitária e tem por missão promover a dignidade humana e a valorização da vida. Busca atuar de modo interdisciplinar e integrado à comunidade acadêmica e à população em geral, desenvolvendo um processo contínuo de ações flexíveis de promoção, prevenção e tratamento dos problemas relacionados ao uso de drogas, e de ensino, pesquisa e formação de recursos humanos.

\section{Organização e processo de trabalho}

O CENPRE trabalha na perspectiva da interdisciplinaridade, contando com profissionais, estagiários e bolsistas de diferentes áreas, tais como psicologia, medicina, enfermagem, serviço social e terapia familiar. Pautado na indissociabilidade entre ensino, pesquisa e extensão, busca uma relação transformadora entre universidade e comunidade.

\section{Atividades de ensino e formação de recursos humanos}

Em 2010 o CENPRE foi contemplado com os recursos do Edital $\mathrm{n}^{\circ}$ 002/2010/GSIPR/SENAD, uma chamada pública para apoio financeiro a projetos de implantação de Centros Regionais de Referência (CRR), voltados para a formação permanente dos profissionais que atuam nas redes de atenção integral à saúde e de assistência social a usuários de crack e outras drogas e seus familiares.

A criação desses CRR foi uma iniciativa da Secretaria Nacional de Políticas sobre Drogas (SENAD) e dos Ministérios da Defesa, da Justiça e da Saúde. Assim, o CENPRE tornou-se um dos 49 centros regionais de formação em recursos humanos que o habilitou para dar início a uma série de cursos de capacitação para os profissionais que atuam na rede assistencial da $3^{\mathrm{a}}$ e da $7^{\mathrm{a}}$ Coordenadorias Regionais de Saúde do Estado do Rio Grande do Sul.

O CRR/FURG foi responsável pelas capacitações de profissionais de muitos municípios da região como, por exemplo, Aceguá, Arroio Grande, Bagé, Candiota, Cerrito, Chuí, Dom Pedrito, Herval, Hulha Negra, Jaguarão, Lavras do Sul, Pedras 
Altas, Pedro Osório, Pinheiro Machado, Rio Grande, Santa Vitória do Palmar e São José do Norte.

Ainda, falando-se em atividades de formação de recursos humanos o CENPRE oferece-se como um cenário para o desenvolvimento de atividades acadêmicas de ensino, a prática de estágios curriculares obrigatórios para os acadêmicos do curso de graduação em Psicologia, estando em programação as atividades para os cursos de Enfermagem e Medicina.

Além disso, o CENPRE possibilita um espaço privilegiado para o processo de aprendizagem, por meio de bolsas de iniciação científica, pesquisa e extensão universitária. Os bolsistas são inseridos em todas as atividades de promoção e prevenção, assistência, pesquisa e formação.

Em nível de pós-graduação, mantém a oferta do Curso de Especialização em Abordagem Multidisciplinar em Dependência Química, já tendo formado oito (08) turmas desde a sua primeira edição (2009). Na realização deste curso participam diferentes unidades acadêmicas da FURG, como o próprio Instituto de Ciências Biológicas (ICB), o Instituto de Educação (IE), o Instituto de Ciências Humanas e da Informação (ICHI), a Faculdade de Medicina (FAMED) e a Escola de Enfermagem (EENF).

\section{Atividades de pesquisa}

Faz parte das atividades do CENPRE desenvolver e participar de projetos de pesquisa relacionados ao tema SPA, auxiliado pelo serviço de banco de dados, com a finalidade de subsidiar cientificamente as intervenções, assim como, contribuir de maneira efetiva na produção de conhecimentos relacionados ao tema.

\section{Atividades de promoção e prevenção}

A tendência dos programas de prevenção é de atuar de forma multifatorial, em que o indivíduo, sua família, a comunidade onde ele está inserido, seu ambiente de trabalho, sua escola, seu ambiente de lazer, de saúde, todos recebem atenção. Dessa forma, um programa de prevenção exige um esforço combinado da comunidade, da escola e das famílias.

As atividades de promoção da saúde e prevenção fizeram parte do trabalho desenvolvido ao longo desses anos, desde o início das atividades do Projeto Drogas, como já foi mencionado. Tais atividades têm o objetivo de dialogar com os diferentes públicos sobre a problemática do uso de SPA. Nestas intervenções são envolvidas escolas, empresas, instituições sociais que acolhem grupos em situação de vulnerabilidade social e diferentes setores públicos.

Dentro desta perspectiva, destaca-se o Projeto De Bem com a Vida (DBV), que prioriza o atendimento de escolares em situação de vulnerabilidade social e a formação de multiplicadores. Desde a sua criação já ofereceu atendimento para mais de 850 jovens. Em 2019, o DBV chegou a sua $42^{\mathrm{a}}$ edição e, por meio de importantes parcerias, possibilitou a formatura de 18 escolares do Projeto Sementes da Esperança, do município de São José do Norte, RS.

\section{Atendimento ambulatorial}

O tratamento da dependência química, complexo e multifatorial, se presta à abordagem integrada e já existe consenso para que seja organizado com enfoque 
interdisciplinar (16). Trabalhar a perspectiva interdisciplinar no cuidado do dependente químico possibilita aprimorar as relações de trabalho entre os profissionais e os pacientes, seus familiares e a própria comunidade. Permite igualmente aproximar os profissionais das necessidades do paciente, proporcionando uma assistência de maior qualidade. A prática do trabalho da equipe interdisciplinar favorece o fortalecimento do vínculo, o acolhimento, a identificação de necessidades e prioridades do atendimento, $o$ acesso aos recursos disponíveis e consequentemente a consolidação do SUS (16).

Os usuários de SPA que buscam os serviços de saúde se dirigem a eles não apenas para interromper o consumo de drogas, mas também para reduzi-lo, minimizar os danos relacionados ao uso, para receber atenção em relação a comprometimentos orgânicos ou psíquicos, construir laços sociais, ter acesso a condições básicas de vida e conquistar autonomia (17).

Observa-se, portanto, a importância do olhar ampliado, da escuta qualificada e da condição de voz dada às pessoas que buscam atendimento, para que sejam considerados nas práticas de saúde o sofrimento e a complexidade da vida dos que chegam aos serviços de saúde (18-19).

Costumeiramente, o encaminhamento ou a procura voluntária decorre do sofrimento ocasionado pela dependência, à própria pessoa e aos seus afetos, diante das consequências danosas praticadas ao corpo e à mente, da possibilidade do diagnóstico de doenças, da exclusão social, violência e mesmo morte.

O CENPRE é um dispositivo que integra a rede de atenção psicossocial do SUS, que trabalha em regime ambulatorial e atende por livre demanda. Toda pessoa pode buscar o serviço com ou sem encaminhamento, estando internado ou não no HU/FURG. Pacientes internados podem ser encaminhados para o CENPRE pelas equipes do hospital e o acolhimento muitas vezes ocorre no leito do paciente, para que seja possível iniciar o vínculo de cuidado antes mesmo da alta hospitalar.

A Política Nacional de Humanização do SUS considera o acolhimento como uma ação que envolve uma escuta qualificada e a capacidade de pactuação entre a demanda do usuário e a possibilidade de resposta do serviço. No CENPRE, o acolhimento é o primeiro contato do paciente e/ou sua família com a equipe, que tem como prioridades a escuta qualificada e a criação de vínculo. Identificadas as expectativas e as necessidades do sujeito acolhido, apresenta-se a estrutura do serviço e o seu funcionamento.

Considerando o contexto hospitalar em que está inserido, o CENPRE atua em conjunto com outros ambulatórios, tais como o de psiquiatria, infectologia, pneumologia, gastrenterologia, Centro de Aplicação e Monitorização de Medicamentos Injetáveis (CAMMI). E atende interconsultas em todas as unidades hospitalares, mantendo diálogo com os serviços de enfermagem, serviço social, medicina, psicologia, entre outros.

Todos os casos atendidos são compartilhados semanalmente pela equipe do CENPRE, que define profissional (is) de referência para cada paciente e discute as possíveis intervenções. Atualmente, as modalidades terapêuticas ofertadas no serviço são os grupos terapêuticos e os atendimentos individuais.

\section{Grupos terapêuticos}

No tratamento da dependência química a abordagem em grupo tem sido utilizada muitas vezes como tratamento de escolha. Esta abordagem é considerada uma intervenção importante e aplicada para usuários de diferentes substâncias (20).

O grupo para dependentes químicos é um instrumento valioso, uma vez que oferece a possibilidade do indivíduo perceber-se parte integrante de um grupo, oferecendo 
alternativas para a solidão e o isolamento. Permite também atender um número maior de pessoas simultaneamente e, por conseguinte, atingir maior número de intervenções compartilhadas, sem desconsiderar os impactos individuais, devidamente facilitados por um mediador.

O CENPRE mantém ativos grupos terapêuticos cuja abordagem tem o objetivo de ajudar a elaborar as dificuldades pessoais. Trata-se a prevenção da recaída, o treinamento de habilidades sociais e a manutenção da abstinência. As relações estabelecidas dentro do grupo fazem com que o indivíduo perceba que não está sozinho e que o grupo irá ajudá-lo no enfrentamento dos seus problemas.

Atualmente, o CENPRE mantém cinco grupos terapêuticos em atividade:

- Em-Frente, que possui o objetivo de fornecer uma nova oportunidade para o crescimento individual, incentivando os participantes ao apoio mútuo e à busca de novas perspectivas para suas vidas, conquistando para si a confiança necessária para perceber sua importância na sociedade.

- Prevenção Terciária, que proporciona um espaço de cuidado e escuta para pacientes que obtiveram alta do tratamento, possibilitando o fortalecimento de suas potencialidades, o compartilhamento de suas dificuldades e contribuindo para a reinserção ou manutenção dos sujeitos em seu meio social.

- Familiar, que tem como objetivo promover um espaço de diálogo com familiares e pessoas próximas dos pacientes, possibilitando trocas de experiências e maior compreensão dos transtornos por uso de substâncias.

- Oficina de Criatividade, para pacientes e familiares, busca produzir relações de cuidado por meio da confecção de trabalhos manuais como ferramenta terapêutica.

- Toque de Expressão, para pacientes e familiares, busca permitir relações de cuidado, tendo a música como instrumento terapêutico.

\section{Atendimento individual}

Após elaboração e avaliação do planto terapêutico, pacientes ou familiares que apresentam necessidade de abordagem individual são encaminhados para atendimento com profissionais da enfermagem, medicina, psicologia, serviço social e/ou para o terapeuta familiar.

\section{O trabalho desenvolvido junto às famílias}

As intervenções com as famílias se constituem em um componente importante quando se consideram as estratégias de intervenção dentro do contexto da dependência (21-23). O impacto que a dependência a drogas gera na vida familiar pode originar a quebra da rotina, sentimentos de vulnerabilidade, desamparo, frustração (24).

A família pode ser considerada como um fator de proteção quando oferece um ambiente harmônico, estável e seguro. Mas ela, a família, pode vir a ser considerada um fator de risco quando este ambiente é desordenado, favorável ao uso de SPA e não oferece nem suporte nem apoio social aos seus membros.

As intervenções feitas com membros de familiares de usuários de álcool e outras drogas ampliam o foco em relação à forma com as famílias vivenciam o problema (24). A abordagem familiar é considerada eficaz e produtiva, pois favorece a adesão do dependente químico ao tratamento, proporciona melhora nos padrões de funcionamento familiar, reduz o consumo, diminui a taxa de recidivas e ajuda na resolução de problemas (25-28). 


\section{O CENPRE em números}

Nos últimos cinco anos (2015 a 2019), o CENPRE realizou ações educativas em estabelecimentos de ensino e empresas privadas, alcançando um público de 1.285 pessoas em Rio Grande e região. Nesse mesmo período, realizou 8.342 atendimentos ambulatoriais, individuais ou em grupos terapêuticos, sendo mais de 3.000 atendimentos somente no ano de 2019. Sua página na internet recebeu mais de um milhão de visitas entre 2015 e 2019.

\section{Considerações finais}

O uso de drogas é considerado uma questão de saúde pública, tanto por aspectos relacionados à prevalência quanto aos agravos e custos à saúde do indivíduo. Desse modo entende-se que é fundamental a articulação das políticas públicas de saúde e dos serviços de assistência e cuidado. De tal modo que a Universidade se coloca como um dispositivo com grande potencial de integrar as diferentes áreas de saber e formação profissional para oferecer o cuidado capacitado à pacientes com demandas tão complexas. Assim como, permite uma integração de atividades de pesquisa, ensino e extensão que culminam, neste caso, em um serviço ambulatorial que presta serviço à comunidade local e região.

Por fim, cabe destacar a importância da continuidade de trabalhos que busquem investigar e relatar as demandas, tanto prevalências quanto fatores associados, atendidas nos serviços com o intuito de se obter subsídios para o planejamento de serviços a essa população. Essa atenção se torna importante para garantir um tratamento que considere o indivíduo em sua integralidade, possibilitando a diminuição dos agravos sociais e de saúde.

\section{Referências}

1 Convention des Nations Unies contre le trafic illicite de stupéfiants et de substances psychotropes 1988. Disponível em: https://www.unodc.org/pdf/convention_1988_fr.pdf

2 Carlini-Cotrim B, Carlini EA, Silva-Filho AR, Barbosa MT. O uso de drogas psicotrópicas por estudantes de primeiro e segundo graus da rede estadual, em dez capitais brasileiras. In: Consumo de drogas psicotrópicas no Brasil, em 1987. Brasília (DF): Centro de Documentação do Ministério da Saúde; 1989. p. 9-84.

3 Brasil. Portaria n 336/2002 de 19 de fevereiro de 2002. Ministério da Saúde. Gabinete do Ministro.

4 Occhini M, Teixeira M. Atendimento a pacientes dependentes de drogas: atuação conjunta do psicólogo e do psiquiatra. Estudos de Psicologia Nata 2006; 11(2): 229-236.

5 Fonseca FN, Gondim APS, Fonteles MMF. Influência dos grupos terapêuticos em Centro de Atenção Psicossocial entre usuários com dependência de cocaína/crack. Saúde Debate 2014; 38(102): 551-561.

6 Brasil. Resolução no 3/GSIPR/CH/CONAD, de 27 de outubro de 2005. Aprova a Política Nacional sobre Drogas. Diário Oficial da União _ 2005; 28 out.

7 Lei $\mathrm{n}^{\mathrm{o}}$ 11.343, de 23 de agosto de 2006. Institui o Sistema Nacional de Políticas Públicas sobre Drogas - Sisnad; prescreve medidas para prevenção do uso indevido, atenção e reinserção social de usuários e dependentes de drogas; estabelece normas para repressão à produção não autorizada e ao tráfico ilícito de drogas; define crimes e dá outras providências.

8 Brasil. Portaria n ${ }^{\circ} 3.088$, de 23 de dezembro de 2011. Institui a Rede de Atenção psicossocial para pessoas com sofrimento ou transtorno mental e com necessidades decorrentes do uso de crack, álcool e outras drogas, no âmbito do Sistema Único de Saúde (SUS). Diário Oficial da União; 2013. 
9 Amarante P, Nunes MO. A reforma psiquiátrica no SUS e a luta por uma sociedade sem manicômios. Ciência e Saúde Coletiva 2018; 23:2067-2074.

10 Trevisan ER, Castro SS. Centros de Atenção Psicossocial - álcool e drogas: perfil dos usuário. Saúde Debate 2019; 43(121): 450-463.

11 Lei 10.872 de 05 de dezembro de 1996. Institui o Conselho Estadual de Entorpecentes - CONEN/RS

12 Lei n. $^{\circ}$ 13.707, de 06 de abril de 2011. Institui o Sistema Estadual de Políticas Públicas sobre Drogas - SEPPED -, o Conselho Estadual de Políticas Públicas sobre Drogas, o Fundo Estadual sobre Drogas - FUNED - e o Departamento Estadual de Políticas Públicas sobre Drogas - DEPPAD -, vinculado à Secretaria da Justiça e dos Direitos Humanos, e dá outras providências.

13 Pietro AT. Drogas: como a legislação brasileira trata do tema? In: Uso de drogas psicoativas. Teoria e métodos para multiplicador prevencionista. Rio Grande, RS; 2012. P133-139.

14 Lei $N^{\circ} 7600$ de 25 de abril de 2014. Institui a política municipal sobre drogas, regulamenta o fundo municipal de política sobre drogas, cria a coordenadoria da política municipal sobre drogas e dá outras providências.

15 Silva FA, Silva ES, Medina J. Histórico do CENPRE. In: Uso de drogas psicoativas. Teoria e métodos para multiplicador prevencionista. Rio Grande, RS; 2012. P29-41

16 Pillon SC, Pegoraro NPJ, Santos MA. O papel da equipe interdisciplinar na dependência química. In: Diehl A, Cordeiro DC, laranjeira R. Dependência química: prevenção, tratamento e políticas públicas. Porto Alegre: Artes Médicas; 2019. p. 455-463.

17 Machado AR, Modena CM, Luz ZMP. O que pessoas que usam drogas buscam em serviços de saúde? Compreensões para além da abstinência. Interface 2020; 24: e190090.

18 Lacerda A, Valla VV. As práticas terapêuticas de cuidado integral à saúde como proposta para aliviar o sofrimento. In: Pinheiro R, Mattos RA. Cuidado: as fronteiras da integralidade. Rio de Janeiro: UERJ, IMS, Abrasco; 2004. p. 93-104.

19 Merhy EE, Feuerwerker LC. Novo olhar sobre as tecnologias de saúde: uma necessidade contemporânea. In: Merhy EE, Baduy RS, Seixas CT, Almeida DES, Slomp Júnior H. Avaliação compartilhada do cuidado em saúde: surpreendendo o instituído nas redes. Rio de Janeiro: Hexis; 2016. p. 59-72.

20 Figlie NB, Lopes da Silva R, Borrego ALS. Psicoterapia de grupo. In: Diehl A, Cordeiro DC, laranjeira R. Dependência química: prevenção, tratamento e políticas públicas. Porto Alegre: Artes Médicas; 2019. p. 285-305.

21 Malbergier A, Cardoso LR, Amaral RA. Uso de substâncias na adolescência e problemas familiares. Cadernos de Saúde Pública 2012; 28(4):678-88.

22 Mangueira SO, Lopes MV. Família disfuncional no contexto do alcoolismo: análise de conceito. Revista Brasileira de Enfermagem 2014; 67(1):149-54.

23 John WT, Gregg ME, Shortt AL, Hutchinson DM, Slaviero TM. Reduction of adolescent alcohol use through family- school intervention: a randomized trial. Adolescent Health 2013; 53(6):778-84.

24 Horta ALM, Daspeti C, Egito JHT, Macedo RMS. Vivências e estratégias de enfrentamento de familiares de dependentes. Revista Brasileira de Enfermagem 2016; 69(6): 962-968.

25 Alvarado $\mathrm{CH}$, Roberto E, Guerra A, Mejías M. Las adolescentes de la calle y su percepción de la sociedad. Enfermeria Global 2013; 12(32): 371-80.

26 Bertrand K, Richer I, Brunelle N, Beaudoin I, Lemieux A, Ménard JM. Substance abuse treatment for adolescents: how are family factors related to substance use change? Journal of Psychoactive Drugs 2013; 45(1): 28-38.

27 Selbekk AS, Sagvaag H, Fausk H. Addiction, families and treatment: a critical realist search for theories that can improve practice. Addiction Research and Theory 2015; 23(3): 196-204.

28 Fatemeh N, Shokouh NN, Reza FM, Va Raftar A. Effectiveness of brief strategic family therapy (bsft) in patterns of family interactions with children involved with drug dependency and relapse prevention. Applied Psychology 2012; 6(24): 29-38. 